Proceedings of the 10th Workshop on Quantum Chaos and Localisation Phenomena (CHAOS 21)

\title{
Always Detectable Eigenfunctions on Metric Graphs
}

\author{
P. KuRASOV* \\ Department of Mathematics, Stockholm Univ., 10691 Stockholm, Sweden \\ Doi: $10.12693 /$ APhysPolA.140.510 \\ *e-mail: kurasov@math.su.se
}

\begin{abstract}
It is proven that Laplacians with standard vertex continuous on metric trees and with standard and Dirichlet conditions on arbitrary metric graphs possess an infinite sequence of simple eigenvalues with the eigenfunctions not equal to zero in any non-Dirichlet vertex.
\end{abstract}

topics: quantum graphs, generic eigenfunctions

\section{Introduction}

The idea of this paper originates from an unpublished work titled "On fully supported eigenfunctions of quantum graphs", 2021, by M. Plümer, M. Täufer [1]. Differential operators on metric graphs attract attention of both mathematicians and physicists due to their unusual but rather elegant spectral properties [2-8]. One such property is that the eigenfunctions may have support not coinciding with the whole graph, or may just vanish at the vertices leading to problems when defining nodal domains. Moreover, if one of the eigenfunctions is vanishing at a vertex $V_{0}$, then it is not seen in the Titchmarsh-Weyl $M$-function associated with this vertex $[4,9]$

$$
M_{V_{0}}(\lambda)=-\left(\sum_{n=1}^{\infty} \frac{\left|\psi_{n}\left(V_{0}\right)\right|^{2}}{\lambda_{n}-\lambda}\right)^{-1}
$$

Here, $\lambda_{n}$ and $\psi_{n}$ are the eigenvalues and orthonormalized eigenfunctions of the operator, respectively. This note is inspired by the recent paper [1] devoted to generic eigenfunctions for metric graphs. By generic eigenfunctions one means the eigenfunctions that are different from zero at all vertices and corresponds to simple eigenvalues. For such eigenfunctions the nodal domains are always clearly defined. It appears that the result can be proven much easier using the language of multivariate secular polynomials describing the spectrum of metric graphs.

\section{Preliminaries}

Let $\Gamma$ be a metric graph formed by $N$ compact edges $E_{n}=\left[x_{2 n-1}, x_{2 n}\right]$ of lengths $l_{n}=x_{2 n}-x_{2 n-1}$ joined together in $M$ vertices $V_{m}$ understood as partitions of the endpoints $\boldsymbol{V}=\left\{x_{j}\right\}_{j=1}^{2 N}$. The points belonging to different equivalence classes $V_{m}$ are identified. In the Hilbert space $L_{2}(\Gamma)=\oplus_{n=1}^{N} L_{2}\left(E_{n}\right) \quad$ consider the Laplacian $L=-\frac{\mathrm{d}^{2}}{\mathrm{~d} x^{2}}$ defined on the functions from the Sobolev space $u \in W_{2}^{2}(\Gamma)=\oplus_{n=1}^{N} W_{2}^{2}\left(E_{n}\right)$, which at every vertex $V_{m}$ satisfy either standard (continuity and Kirchhoff) conditions, i.e.,

$$
\left\{\begin{array}{l}
u \text { is continuous at } V_{m}, \\
\sum_{x_{j} \in V_{m}} \partial u\left(x_{j}\right)=0
\end{array}\right.
$$

or Dirichlet conditions

$$
u\left(x_{j}\right)=0, \quad x_{j} \in V_{m} .
$$

The directed derivatives $\partial u\left(x_{j}\right)=-(-1)^{j} u^{\prime}\left(x_{j}\right)$ are taken in the direction pointing inside the corresponding edge. We assume that all vertices with Dirichlet conditions have degree one and we call them Dirichlet vertices. All other vertices are called standard. The Laplacian is a non-negative self-adjoint operator with discrete spectrum. It is uniquely determined by the metric graph $\Gamma$, provided the Dirichlet vertices are indicated. In what follows we are going to refer to its spectrum as graph's spectrum.

Any solution to the eigenfunction equation on the edge $E_{n}$ can be written using one of the two equivalent representations

$$
\begin{aligned}
& \psi(x, \lambda)= \\
& a_{2 n-1} \mathrm{e}^{-\mathrm{i} k\left|x-x_{2 n-1}\right|}+a_{2 n} \mathrm{e}^{-\mathrm{i} k\left|x-x_{2 n}\right|}= \\
& b_{2 n-1} \mathrm{e}^{\mathrm{i} k\left|x-x_{2 n-1}\right|}+b_{2 n} \mathrm{e}^{\mathrm{i} k\left|x-x_{2 n}\right|} .
\end{aligned}
$$

Introducing the $2 N$-dimensional vectors, $\boldsymbol{a}=\left\{a_{j}\right\}_{j=1}^{2 N}$ and $\boldsymbol{b}=\left\{b_{j}\right\}_{j=1}^{2 N}$, one gets two linear relations $\boldsymbol{b}=S_{\mathrm{e}}(k) \boldsymbol{a}, \boldsymbol{b}=S_{\mathrm{v}} \boldsymbol{a}$ with

$$
S_{\mathrm{e}}(k)=\operatorname{diag}\left[\left(\begin{array}{cc}
0 & \mathrm{e}^{\mathrm{i} k l_{n}} \\
\mathrm{e}^{\mathrm{i} k l_{n}} & 0
\end{array}\right)\right]_{n=1}^{N}
$$


and $S_{\mathrm{v}}$ formed from the vertex scattering matrices $S_{m}, m=1,2, \ldots, M$ given by

$$
S_{m}=S_{d_{m}}^{\text {st }}=-\mathbb{I}_{d_{m}}+\frac{d_{m}}{2}\left(\begin{array}{cccc}
1 & 1 & \ldots & 1 \\
1 & 1 & \ldots & 1 \\
\vdots & \vdots & \ddots & 1 \\
1 & 1 & \ldots & 1
\end{array}\right)
$$

and

$$
S_{m}=S^{D}=-1
$$

for standard vertices of degree $d_{m}$ and for Dirichlet vertices, respectively. The vertex scattering matrix $S_{\mathrm{v}}$ has a block-diagonal structure if one permutes the endpoints collecting together the points belonging to each of the vertices. The first relation comes directly from (4) and the second one is obtained by substituting solutions (4) into vertex conditions (2) and (3).

The positive spectrum $\lambda=k^{2}$ can be described as the zeroes of the secular function $[4,10,11]$

$$
p(k):=\operatorname{det}\left(S_{\mathrm{e}}(k)-S_{\mathrm{v}}\right),
$$

which is a trigonometric polynomial.

Following $[5,12]$ let us introduce the secular polynomials in $N$ complex variables $\boldsymbol{z}=\left(z_{1}, z_{2}, \ldots, z_{N}\right)$ in the form of

$$
\begin{aligned}
& P_{G}(\boldsymbol{z})=\operatorname{det}\left(E(\boldsymbol{z})-S_{\mathrm{v}}\right), \\
& E(\boldsymbol{z})=\operatorname{diag}\left[\left(\begin{array}{cc}
0 & z_{n} \\
z_{n} & 0
\end{array}\right)\right]_{n=1}^{N},
\end{aligned}
$$

so that we have

$$
p(k)=P\left(\mathrm{e}^{\mathrm{i} k \boldsymbol{l}}\right), \quad \boldsymbol{l}=\left(l_{1}, l_{2}, \ldots, l_{n}\right) .
$$

Note that the secular polynomial $P_{G}$ is determined by the discrete graph $G$ corresponding to $\Gamma$ and by the set of Dirichlet vertices, but is independent of the metric structure of $\Gamma$. The spectrum of the metric graph is given by the intersections of the curve $\mathrm{e}^{\mathrm{i} k l}$ with the zero set $\mathrm{Z}_{G}$ of the secular polynomial. Thus,

$$
\mathbb{Z}_{G}=\left\{\boldsymbol{z} \in \mathbb{T}^{N} \subset \mathbb{C}^{N}: P(\boldsymbol{z})=0\right\},
$$

where $\mathbb{T}=\{z \in \mathbb{C}:|z|=1\}$ is the unit torus. We shall also use real coordinates $\varphi$ and $z_{n}=\mathrm{e}^{\mathrm{i} \varphi_{n}}$, then the spectrum is given by intersections of the line $k \boldsymbol{l}$ and the zero set

$$
\mathrm{Z}_{G}=\left\{\boldsymbol{\varphi} \in \mathrm{T}^{N}: P\left(\mathrm{e}^{\mathrm{i} \varphi}\right)=0\right\},
$$

with $\mathrm{T}=[0,2 \pi]$ being the real torus. The zero set $\mathrm{Z}_{G}$ in general is an $(N-1)$-dimensional singular surface on the torus.

\section{Main theorem}

Definition 1. An eigenvalue $\lambda_{n}$ and the corresponding eigenfunction are called generic if and only if

1. the eigenvalue is simple,

2. the corresponding eigenfunction does not vanish in any of the vertices other than at the Dirichlet vertices.
Theorem 1. (Theorems 1 and 2 from [1]). Let $L(\Gamma)$ be the Laplace operator on a finite compact metric graph $\Gamma$ with standard and Dirichlet vertex conditions. Then there exists an infinite sequence of generic eigenfunctions attaining positive values at the vertices, provided that either

- the graph is a tree,

or

- the set of Dirichlet vertices is non-empty.

Proof. It is well-known that the order of positive zeroes of the secular function $p(k)$ coincides with the multiplicity of the corresponding eigenvalues $[2,4]$. Hence to satisfy the first genericness condition (in Definition 1), it is sufficient that the line $k \boldsymbol{l}$ crosses $\mathrm{Z}_{G}$ at a regular point.

Assume first that the graph has a Dirichlet vertex, then the line $k \boldsymbol{l}, k>0$, for any choice of the vector $\boldsymbol{l}$ crosses first the zero set $\mathrm{Z}_{G}$ at a regular point $\varphi^{1}=\varphi^{1}(\boldsymbol{l})$. The eigenvalue $\lambda^{1}=\left(k^{1}\right)^{2}$ is determined by $k^{1} \boldsymbol{l}=\boldsymbol{\varphi}^{1}$. The ground state is always simple (for connected graphs) [14] and the corresponding eigenfunction can be chosen strictly positive, that is positive everywhere except at the Dirichlet vertices. Moreover its dependence on the edge lengths is described by Hadamard-type formula (see e.g. Eq. (3.13) in [14] following [15-17]

$$
\frac{\mathrm{d} \lambda_{1}}{\mathrm{~d} l_{n}}=-\left.\left(\psi_{1}^{\prime}(x)^{2}+\lambda_{1} \psi_{1}(x)^{2}\right)\right|_{x \in E_{n}}
$$

connecting the derivative of the ground state energy $\lambda_{1}$ as the length of the edge $E_{n}$ changes to Prüfer's amplitude [1] of the eigenfunction $\psi_{1}$ on the edge. The derivatives above are zero if and only if $\psi_{1}^{\prime}(x)=0$ and $\lambda_{1}=0$, that is if the ground state is a constant function, which is not the case if Dirichlet vertices are present. It follows that $\nabla P\left(\boldsymbol{z}^{1}\right) \neq \mathbf{0}$, $\boldsymbol{z}^{1}=\mathrm{e}^{\mathrm{i} \varphi^{1}}$, and this inequality holds in a neighbourhood of $\boldsymbol{z}^{1}$. Independently of whether the edge lengths are rationally dependent or not the crossing points between $k \boldsymbol{l}$ and $\mathrm{Z}_{G}$ contain an infinite sequence $\varphi^{j}$ tending to $\varphi^{1}$ :

- if the edge lengths are pairwise rationally dependent, then the line $k \boldsymbol{l}$ passes $\varphi^{1}$ infinitely many times;

- if the edge lengths are not pairwise rationally dependent, then the intersection points do not coincide with $\varphi^{1}$, but approaches it as $j \rightarrow \infty$.

As $\quad \varphi^{j} \stackrel{j \rightarrow \infty}{\longrightarrow} \varphi^{1}$, the corresponding vector $\boldsymbol{a}=\boldsymbol{a}\left(\boldsymbol{\varphi}^{j}\right)$ solving

$$
\left[E\left(\mathrm{e}^{\mathrm{i} k \boldsymbol{\varphi}^{j}}\right)-S_{\mathrm{v}}\right] \boldsymbol{a}=0
$$

converges to $\boldsymbol{a}\left(\boldsymbol{\varphi}^{1}\right)$. Similarly

$$
\boldsymbol{b}\left(\varphi^{j}\right)=S_{\mathrm{v}} \boldsymbol{a}\left(\varphi^{j}\right) \stackrel{j \rightarrow \infty}{\longrightarrow} S_{\mathrm{v}} \boldsymbol{a}\left(\varphi^{1}\right)=\boldsymbol{b}\left(\varphi^{1}\right) .
$$

It follows that

$$
\psi_{\lambda^{j}}\left(x_{i}\right)=a_{i}\left(\varphi^{j}\right)+b_{i}\left(\varphi^{j}\right) \stackrel{j \rightarrow \infty}{\longrightarrow} \psi_{\lambda^{1}}\left(x_{i}\right) \neq 0
$$




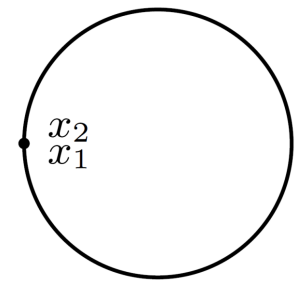

Fig. 1. The circle graph.

where $\lambda^{j}=\left(k^{j}\right)^{2}$ denotes the eigenvalue associated with the crossing point $\varphi^{j}=k^{j} l$ and provided $x_{j}$ does not belong to a Dirichlet vertex. It follows that, may be taking a subsequence, all eigenvalues $\lambda^{j}$ are not only simple, but the corresponding eigenfunctions are different from zero at non-Dirichlet vertices.

It remains to consider the case where $\Gamma$ is a tree with only standard vertex conditions. In the proof above it was sufficient to have one generic eigenvalue such that the associated crossing point is a regular point in the zero set $\mathrm{Z}_{G}$. One possible candidate is the ground state $\lambda_{1}=0$ and the eigenfunction identically equal to 1 . The corresponding Prüfer amplitude is zero and we cannot conclude that the point $\boldsymbol{\varphi}=\mathbf{0}$ is a regular point in $\mathrm{Z}_{G}$. Consider the corresponding equilateral tree $\mathrm{T}$ with all edge lengths equal to $l_{n}=l$. The spectrum is then periodic with period $\frac{2 \pi}{l}$. Consider the eigenfunction associated with the eigenvalue $\left(\frac{2 \pi}{l}\right)^{2}$, its multiplicity is equal to 1 [18]. ${ }^{\dagger}$ This function attains 1 at all vertices (the same value as the ground state eigenfunction) and the corresponding eigenvalue is non-zero, hence Prüfer amplitudes are different from zero. It follows that the point $\varphi=(2 \pi, 2 \pi, \ldots, 2 \pi)$ and hence the point $\boldsymbol{\varphi}=\mathbf{0}$ is a regular point in $\mathrm{Z}_{G}$. It remains to repeat the arguments used in the first part of the proof, i.e., for any choice of the edge lengths there is a sequence $\varphi^{j}$ of intersection points between $k \boldsymbol{l}$ and $\mathrm{Z}_{G}$ approaching $\boldsymbol{\varphi}^{1}=\mathbf{0}$. The corresponding eigenvalues are simple and the eigenfunction values at each vertex approach 1. Taking a subsequence, if necessary, we get an infinite sequence of generic eigenvalues.

\section{Conclusions}

In our opinion the new proof explains the reason why conditions in the theorem are needed. Our proof is based on the existence of a single generic eigenfunction. The ground state eigenfunction is a good candidate since we know that it

\footnotetext{
${ }^{\dagger 1}$ The multiplicity of the eigenvalue $\left(\frac{2 \pi}{l}\right)^{2}$ for equilateral graphs in general is equal to $1+\beta_{1}$, where $\beta_{1}$ is the first Betti number - the number of cycles in a graph. In the case of trees $\beta_{1}=0$.
}

is always generic. The proof goes well for graphs with Dirichlet points, but for graphs with standard conditions one needs to prove that the hypersurface $\mathrm{Z}_{G}$ is regular in a neighbourhood of 0 . If the graph has cycles, then the point 0 is not regular $\nabla P_{G}(1)=0$. It might happen that $P_{G}$ is a square of the first degree polynomial like in the case of the cycle graph $P\left(z_{1}\right)=\left(z_{1}-1\right)^{2}$, then the set $\mathrm{Z}_{\mathrm{G}}$ is a smooth surface, but all non-zero eigenvalues are double.

In fact the circle graph (see Fig. 1) provides a counterexample as it is impossible to find an infinite sequence of simple eigenvalues.

The theorem has another interesting implication. Under conditions of Theorem 1 there always exists an infinite sequence of simple eigenvalues with an even/odd number of nodal domains, provided the Euler characteristic $\chi=M-N$ is even/odd, respectively.

\section{References}

[1] M. Plümer, M. Täufer, arXiv:2106.10096, 2021.

[2] G. Berkolaiko, P. Kuchment, Introduction to quantum graphs, Mathematical Surveys and Monographs, Vol. 186, American Mathematical Society, Providence (RI) 2013.

[3] O. Hul, S. Bauch, P. Pakoński, N. Savytskyy, K. Życzkowski, L. Sirko, Phys. Rev. E 69, 056205 (2004).

[4] P. Kurasov, Spectral Geometry of Graphs, Birkhäuser, 2021.

[5] P. Kurasov, P. Sarnak, J. Math. Phys. 61 , 083501 (2020).

[6] M. Ławniczak, P. Kurasov, Sz. Bauch, M. Białous, V. Yunko, L. Sirko, Phys. Rev. E 101, 052320 (2020).

[7] M. Ławniczak, P. Kurasov, S. Bauch, M. Białous, A. Akhshami, L. Sirko, Sci. Rep. E 11, 15342 (2021).

[8] M. Ławniczak, P. Kurasov, S. Bauch, M. Białous, L. Sirko, Acta Phys. Pol. A 139, 323 (2021).

[9] P. Kurasov, Acta Phys. Pol. A 132, 1666 (2017).

[10] B. Gutkin, U. Smilansky, J. Phys. A 34 , 6061 (2001).

[11] P. Kurasov, M. Nowaczyk, J. Phys. A 38, 4901 (2005).

[12] F. Barra, P. Gaspard, J. Statist. Phys. 101, 283 (2000).

[13] P. Kurasov, Lett. Math. Phys. 109, 2491 (2019).

[14] G. Berkolaiko, J.B. Kennedy, P. Kurasov, D. Mugnolo, Trans. Amer. Math. Soc. 372 , 5153 (2019). 
[15] R. Band, G. Lévy, Guillaume, Ann. Henri Poincaré 18, 3269 (2017).

[16] Y. Colin de Verdiére, Ann. Henri Poincaré 16, 347 (2015).

[17] L. Friedlander, Israel J. Math. 146, 149 (2005).

[18] P. Kurasov, J. Funct. Anal. 254, 934 (2008). 